\title{
Evidence for adaptation of porcine Toll-like receptors
}

\author{
Kwame A. Darfour-Oduro ${ }^{1} \cdot$ Hendrik-Jan Megens ${ }^{2} \cdot$ Alfred Roca $^{1}$ • \\ Martien A. M. Groenen ${ }^{2}$ - Lawrence B. Schook ${ }^{1}$
}

Received: 6 October 2015 / Accepted: 14 December 2015 /Published online: 23 December 2015

(C) The Author(s) 2015. This article is published with open access at Springerlink.com

\begin{abstract}
Naturally endemic infectious diseases provide selective pressures for pig populations. Toll-like receptors $(T L R S)$ represent the first line of immune defense against pathogens and are likely to play a crucial adaptive role for pig populations. This study was done to determine whether wild and domestic pig populations representing diverse global environments demonstrate local TLR adaptation. The genomic sequence encoding the ectodomain, responsible for interacting with pathogen ligands of bacterial (TLR1, TLR2 and TLRO) and viral (TLR3, TLR7 and TLR8) receptors, was obtained. Mitochondrial D-loop region sequences were obtained and a phylogenetic analysis using these sequences revealed a clear separation of animals into Asian $(n=27)$ and European $(n=40)$ clades. The TLR sequences were then analyzed for population-specific positive selection signatures within wild boars and domesticated pig populations derived from Asian and European clades. Using within-population and betweenpopulation tests for positive selection, a TLR2-derived variant 376A (126Thr), estimated to have arisen in 163,000 years ago with a frequency of $83.33 \%$ within European wild boars, $98.00 \%$ within domestic pig breeds of European origin, $40.00 \%$ within Asian wild boars, and $11.36 \%$ within Asian domestic pigs, was identified to be under positive selection in
\end{abstract}

Electronic supplementary material The online version of this article (doi:10.1007/s00251-015-0892-8) contains supplementary material, which is available to authorized users.

Lawrence B. Schook schook@uillinois.edu

1 Department of Animal Sciences, University of Illinois, Urbana-Champaign, IL 61801, USA

2 Animal Breeding and Genomics Centre, Wageningen University, Droevendaalsesteeg 1, Wageningen 6708 PB, The Netherlands pigs of European origin. The variant is located within the $\mathrm{N}$ terminal domain of the TLR2 protein $3 \mathrm{D}$ crystal structure and could affect ligand binding. This study suggests the TLR2 gene contributing to responses to bacterial pathogens has been crucial in adaptation of pigs to pathogens.

Keywords TLR $\cdot$ Porcine $\cdot$ Local adaptation $\cdot$ Immunity Infectious diseases

\section{Introduction}

European and Asian wild boars diverged 1.6-0.8 million years (Myr) ago (Frantz et al. 2013) with domestication events occurring approximately 9000 years ago (Giuffra et al. 2000). European wild boars and European domesticated pigs and their Asian counterparts may have experienced different selective pressures given that they have originated from different continents with different endemic infectious diseases (Troy et al. 2001; Novembre and Di Rienzo 2009). Such differences in selective pressures can be exploited to detect immune-related genes that have been of adaptive value in terms of disease resistance within pig populations.

The vertebrate immune system is comprised of the innate and acquired immune components. The innate immune component is ancient and characterized by rapid and non-specific responses to pathogenic microbes while the acquired immune component is characterized by highly specific memory responses. The Toll-like receptor (TLR) family represents a class of innate immunity receptors important during early phases of infections that also serve as a link between the innate and acquired immune responses (Werling and Jungi 2003), consequently triggering inflammatory responses to prevent bacterial and viral spread. In vertebrates, 10-12 TLRs have been identified (Leulier and Lemaitre 2008) and are classified based on 
their cellular location and the type of pathogenic ligands they recognize. Cell surface expressed TLRS (TLR1, TLR2, TLR4, $T L R 5$, and TLRO) recognize predominantly bacterial ligands and several fungal and parasite ligands while TLR3,TLR7, $T L R 8$, and TLR 9 are expressed within endosomes and recognize single- and double-stranded RNA and CpG DNA (Akira et al. 2006). Single nucleotide polymorphisms (SNPs) and differences in expression of TLR molecules affect immune responses to numerous pathogens and are associated with host resistance to infectious diseases (Lazarus et al. 2002). Several nonsynonymous substitutions have been detected in porcine $T L R$ genes, especially in the ectodomain of cell surface-expressed TLRs (Uenishi et al. 2011), suggesting functional relevance of the ectodomain and specific SNPs residing within these regions.

Naturally occurring porcine diseases endemic to particular geographic locations include African swine fever prevalent in sub-Saharan Africa; the ancient foot and mouth disease endemic in some regions of Asia, most of Africa, and the Middle East; brucellosis observed in the Middle East, the Mediterranean region, sub-Saharan Africa, China, India, Peru, and Mexico; and swine influenza which is endemic to North and South America, Europe, and parts of Asia (OIE 2012). The occurrence of such locally or regionally endemic diseases suggests that long-term adaptation may have occurred between the host and pathogen (Zimmerman et al. 2012). Thus, such adaptation could be detected as genomic signatures across populations. Recent studies have shown that approximately $7 \%$ of the pig genome has been influenced by selection either in the form of positive or balancing selection (Amaral et al. 2011). These signals were observed in genomic regions involved in coat color, brain and muscle development, growth, metabolism, olfaction, and immunity and were specific to certain pig populations (Amaral et al. 2011). Differences in amino acid substitutions and heterozygosity of TLR SNPs in European and Asian pigs have been observed and might be due to differences in pathogens encountered in the domestication and initial breed development environments of the pigs (Shinkai et al. 2006). Porcine TLRs therefore represent candidate immune genes for investigating pathogen-driven selective pressures specific to geographically defined populations.

The aim of this study was to determine whether adaptation to local environments of wild and domestic European and Asian pig populations resulted in $T L R$ gene genomic signatures of selection. We hypothesized that geographically restricted selective pressures resulted in adaptive genomic signatures within porcine TLRs. To test this hypothesis, the sequences encoding the ectodomain of bacterial recognizing (TLR1, TLR2, and TLRO) and viral sensing (TLR3, TLR7, and $T L R 8)$ receptors were obtained and the following specific questions were addressed: (1) whether adaptive selection pressures on pigs from both European and Asian environments was evident and (2) whether different selective pressures for each geographic environment were identified.

\section{Materials and methods}

\section{Study populations}

Sixty-seven individuals consisting of wild boars and domestic pigs representing populations with origins from diverse geographic environments within Asia and Europe were utilized in this study. European wild boars were represented by 11 unrelated individuals from the Netherlands, 2 from Greece, and 1 each from Switzerland and Italy. European domestic breeds were sampled from unrelated pigs including Angler Sattleschwein (1), Mangalitsa (1), Durocs (4), Hampshires (3), Landraces (6), Pietrains (4), Charto Murciano (2), Cassertana (2), Retinto (1), and Cinta Senese (1). Asian wild boars were represented by individuals from Northern China (3), from Southern China (1), and from Japan (1). Finally, Asian domestic pigs included Meishan pigs (10), Jiangquahai pigs (3), Xiang pigs (2), Wannan spotted pigs (2), Jinhua pigs (2), Leping spotted pigs (2), and Zang pig (1). Animals within a group were not related (no shared grandparents). In addition, one species individual from the family Suidae (Phacochoerus africanus (Common warthog), Sus verrucosus (Javan warty pig), or Sus celebensis (Sulawesi warty pig) were also analyzed. The source of DNA samples have been previously described (Bosse et al. 2012). Supplementary Fig. S1 shows the geographic sampling regions.

\section{Loci analyzed}

Six TLR genes chosen for this study are described in Supplementary Table S1. The TLRs ectodomains were examined since they are the functional sites involved in pathogen ligand recognition. The D-loop region of mtDNA, a neutral marker, was used to demonstrate the independent domestication origins of European and Asian pigs (Larson et al. 2005). The Dloop region of the mtDNA of each animal was obtained by direct sequencing and analyzed to obtain clustering patterns among the sampled animals. On each of the 18 autosomal chromosomes of pigs, one noncoding genomic region $(2 \mathrm{~kb}$ in size) (Supplementary Table S2) was chosen to represent neutrally evolving regions. These noncoding genomic regions were at least $50 \mathrm{~kb}$ from any known or predicted porcine gene, as determined by inspecting the Sus scrofa 10.2 genome assembly on the UCSC genome browser. The $50-\mathrm{kb}$ buffer was chosen to minimize the effect of linkage disequilibrium with genic SNPs (Wagh et al. 2012).

\section{DNA sequencing}

DNA extraction, library preparation, and sequencing have been described previously (Bosse et al. 2012). Briefly, DNA 
was extracted from whole blood by using the QIAamp DNA blood spin kit (Qiagen Sciences), and quantity and quality parameters were performed on the Qubit 2.0 fluorometer (Invitrogen) and run on a $1 \%$ agarose gel. Library construction and re-sequencing of individual pig samples utilized 1$3 \mu \mathrm{g}$ of genomic DNA following Illumina library preparation protocols. The library insert size was 300-500 bp and sequencing was performed using the 100 paired-end sequencing kit. All DNA were sequenced to approximately $8 \times$ depth. Quality trimmed reads (phred quality $>20$, minimum length of pairs of reads $=40 \mathrm{bp}$ ) were aligned to the $S$. scrofa reference genome build 10.2 using the unique alignment option of Mosaik Aligner (V.1.1.0017). The aligned reads from each of the animals together with the $S$. scrofa reference genome were stored as bam files for each individual animal.

The coordinates of genes within the bam files were obtained by querying the $S$. scrofa reference genome build 10.2 with coding sequences of porcine TLRs from public databases. The accession numbers of sequences obtained from the public databases for TLRs were TLR1: NM_001031775, TLR2: NM_213761, TLR3: HQ412796, TLR6: NM_213760, TLR7: NM_001097434, and TLR8: ENSSSCG00000012118. Perl scripts were used to extract the TLR coding sequences and the noncoding genomic regions. Sequences were aligned using ClustalW 1.81 (Thompson et al. 1997). The D-loop region of mtDNA was obtained by direct sequencing.

A 715-bp fragment of the D-loop region was amplified by polymerase chain reaction (PCR). The nucleotide sequences of the primers used were as follows: forward 5' CTCCGCCATCAGCACCCAAAG3' and reverse 5' GCACCTTGTTTGGATTRTCG3' (Luetkemeier et al. 2010). Final reactions were made to a final volume of $12 \mu \mathrm{L}$ containing each reaction PCR master mix (Thermo Fisher Scientific, USA) which supplies $1.5 \mathrm{mM} \mathrm{MgCl}_{2}, 0.625 \mathrm{U}$ of Taq DNA polymerase, and $0.2 \mathrm{mM}$ dNTPs. Then, $5 \mathrm{pM}$ of each primer and $\sim 40$ ng DNA were added to the final reaction. Amplification protocol was performed as follows: 35 cycles, each consisting of $95^{\circ} \mathrm{C}$ for $30 \mathrm{~s}, 55^{\circ} \mathrm{C}$ for $45 \mathrm{~s}$, and $72^{\circ} \mathrm{C}$ for $90 \mathrm{~s}$ and then a final extension at $72{ }^{\circ} \mathrm{C}$ for $10 \mathrm{~min}$. The amplicon was confirmed using $2 \mu \mathrm{L}$ PCR product by electrophoresis on a $1.5 \%$ agarose gel stained with ethidium bromate visualized under UV light. The remaining PCR products were purified using a Multiscreen PCR 96 cleanup vacuum system (Millipore). Bidirectional sequencing reactions were carried out using Big-Dye Terminator Cycle Sequencing Ready Reaction kit v3.1 (Applied Biosystem, USA) in an ABI3730 DNA Analyzer sequencer (Applied Biosystem, USA). In order to correct sequencing errors and obtain the consensus sequence for each individual amplicon, GAP 4 was utilized (Bonfield et al. 1995), using the mtDNA pig sequence GenBank AJ002189 (Ursing and Arnason 1998) as a reference sequence.

\section{Data analysis}

Porcine TLR reference amino acid sequences were aligned to the corresponding human sequences in order to delineate the ectodomain, the leucine-rich repeat (LRR) modules, ligandbinding and dimerization domains, and other sub-domains within the ectodomain (Supplementary Fig. S2). Haplotype reconstructions from the aligned sequences for all loci were carried out with PHASE 2.1.1 software (Stephens and Scheet 2005) using the SNiPLAY web-based tool for SNP and polymorphism analysis (Dereeper et al. 2011). For the D-loop region sequences of mtDNA, the Neighbor-Joining method (using p-distance) implemented in MEGA version 5 (Tamura et al. 2011) was used for phylogenetic analysis (Supplementary Fig. S3). A bootstrap of 1000 replicates was conducted. To test for departure from the standard neutral model of evolution, Tajima's D, Fu and Li's D*, and Fu and Li's F* were conducted using DnaSP (Librado and Rozas 2009).

Derived allele under positive selection was determined using the derived intrallelic nucleotide diversity (DIND) test. The DIND test was applied by plotting for all SNPs within the ectodomain of genes within groups that showed deviation from neutrality, the ratio between the ancestral and derived internal nucleotide diversity (diversity among haplotypes carrying alleles) against the frequencies of derived alleles. An elevated ratio associated with a high derived allele frequency was used as an indication of positive selection of the derived allelic state. To define statistical significance, the values estimated for TLRs were then compared against the background neutral distribution obtained by means of 10,000 simulations of the sequences encoding the ectodomain conditional on the number of segregating sites and the recombination rate of the sequences and integrating a simplified version of a wild boar demographic model (initial effective population sizes of European and Asian wild boars $=\sim 25,000$, a bottleneck at 20, 000 years ago, and an effective population size 10,000 years ago of 3000 for European wild boars and 13,500 for Asian wild boars) (Groenen et al. 2012). Simulations were carried out using Fastsimcoal2 (Excoffier et al. 2013). TLR SNPs that fell beyond 90th and 95th percentiles of the neutral distribution were considered to be under positive selection. Singletons were excluded from this analysis.

Interpopulation differentiation $\left(\mathrm{F}_{\mathrm{ST}}\right)$ and expected loci heterozygosity were calculated for TLR SNPs that were polymorphic in each of two populations being compared and intergenic region sequences SNPs using Arlequin ver 3.5 (Excoffier and Lischer 2010). TLR SNPs showing high levels of population differentiation and, thus, the target of positive selection were identified by comparison of TLR SNP $\mathrm{F}_{\mathrm{ST}}$ values and the 90th and 95th percentiles for $\mathrm{F}_{\mathrm{ST}}$ distribution (estimated using heterozygosity sliding windows of size 0.025 with increasing step of 0.01) computed for SNPs of the noncoding genomic regions. The $p$ value for a SNP was estimated 
(Barreiro et al. 2009) where, first, $\mathrm{F}_{\mathrm{ST}}$ values for a TLR SNP was compared with $\mathrm{F}_{\mathrm{ST}}$ values from the noncoding genomic region sequences SNPs with an expected heterozygosity value of \pm 0.025 with respect to that observed for the TLR SNP. Then among the noncoding genomic region SNPs, the proportion of SNPs with $\mathrm{F}_{\mathrm{ST}}$ values higher than that observed for the $T L R$ SNP was used as the $p$ value. Ancestral and derived states of $T L R$ alleles were determined by a strategy (Groenen et al. 2012) where an allele is assumed to be ancestral when one of the alleles in $S$. scrofa was observed in P. africanus (Common warthog), S. verrucosus (Javan warty pig), or $S$. celebensis (Sulawesi warty pig) in that order, respectively. A maximum likelihood approach implemented in GENETREE version 9 (Griffiths and Tavare 1994) was used to estimate theta $\left(=4 \mathrm{~N}_{\mathrm{e}} \mu\right)$ and age of mutations. The default mutation rate $\left(\mu=2.5 \times 10^{-8}\right)$ of humans was used as there is no known mutation rates for pigs (Groenen et al. 2012). Time estimated in generations $(T)$ were converted into years $(t)$ using a 5 -year generation time $(g)$ with the formula $t=2 \times N_{\mathrm{e}} \times T \times g$ as stated in the GENETREE manual. Median-joining phylogenetic haplotype networks were constructed based on SNPs within the TLRs using Network 4.6.1.1 (www.fluxus-technology. com). Only haplotypes present in a minimum of two animals were considered. MuPIT Interactive (Niknafs et al. 2013) was used to map variants under selection on to three-dimensional (3D) protein structures. Swiss prot and Ensemble genome browsers were utilized to determine the functional consequences of TLR sites under positive selection.

\section{Results}

Sequences (67 sequences for each TLR alignment) encoding the ectodomains of bacterial sensing TLR1, TLR2, and TLR6 and viral sensing $T L R 3, T L R 7$, and TLR8 from wild boars and domestic pigs of European and Asian origins were obtained. The length of the sequences in terms of number of nucleotides of the TLRs ranged from 1668 bases for TLRI to 2445 bases for TLR7. Amino acid length ranged from 556 amino acids for TLR1 to 792 amino acids for TLR7. A total of 136 SNPs were identified within the TLR sequences encoding the ectodomain (Supplementary Table S3). Majority of the SNPs have been previously reported (Shinkai et al. 2006; Morozumi and Uenishi 2009; Bergman et al. 2010).

\section{Evidence of positive selection pressure within population}

To determine whether there is evidence of positive selective pressure mediated by infectious agents of endemic diseases on pig populations, wild boars and domestic pigs from previously defined European and Asian lineages were chosen (Megens et al. 2008). The geographic origins of these populations were validated by sequencing the mitochondrial D-loop regions of these animals and constructing a phylogenetic tree. The Neighbor Joining tree (Supplementary Fig. S3) obtained from analysis of the D-loop region sequences revealed two clades of animals representing animals of European and Asian origins. For analysis, animals were grouped by geographic origins and domestication status. The groups considered were therefore all European animals (wild boars and domestic pigs combined, $N=40$ ), all Asian animals (wild boars and domestic pigs combined, $N=27)$, Asian wild boars $(N=5)$, European wild boars $(N=15)$, Asian domestic pigs $(N=22)$, and European domestic pigs $(N=25)$. Gene sequences of bacterial sensing TLR1, TLR2, and TLR6 and viral sensing TLR3, $T L R 7$, and $T L R 8$ for each animal were extracted from whole genome resequenced data from each animal. Analysis of positive selection focused on the ectodomains (Supplemetary Table S1) involved in pathogen recognition.

The following tests were performed to determine evidence of adaptive selection pressure for European and Asian pig populations: (1) test of deviation from neutrality (sliding window analysis of Tajima's D, Fu and Li's D*, and Fu and Li's $\mathrm{F}^{*}$ ) due to shift to a low frequency spectrum polymorphism; and (2) test for derived alleles under recent positive selection due to high frequency of the allele in a population (Rubin et al. 2012) using the DIND test (Barreiro et al. 2009). Given the relatively small population sample sizes, genes under selection were defined conservatively as those for which both neutrality and DIND test were significant in the same population (Manry et al. 2011). Using this stringent criteria, TLRS (TLR1, TLR3, TLR6, TLR7, and TLR8) investigated in this study did not show signatures of adaptive selection (data not shown) in any population. The stringent criteria employed here and the limited number of genes used in this study indicated that fewer number of SNPs were expected to show signatures of adaptive selection. Each of the three neutrality tests detected significant $(p<0.05)$ excess of rare alleles within the bacterial sensing TLR2 exon 2 (encoding the ectodomain) of the European (wild boars and domestic pigs combined) population (Tajima's $\mathrm{D}=-1.80 ; \mathrm{Fu}$ and Li's $\mathrm{D}^{*}=-3.74$; Fu and Li's $\mathrm{F}^{*}=-3.67$, Fig. 1) consistent with positive selection or population expansion. Based on the DIND test involving SNPs within the TLR2 sequences of the European population, the derived allele TLR2 SNP 376A (126Thr), located on exon 2 of the TLR2 gene with a frequency of $92.5 \%$ (83.33\% within European wild boars and $98.00 \%$ within domestic pig breeds of Europe), was detected as showing evidence of positive selection $(\pi \mathrm{A} / \pi \mathrm{D}=6.88 ; p=0.055$, Fig. 2). Three European wild boars were heterozygous, 1 wild boar was homozygous for the ancestral allele, whereas the 11 remaining wild boars were homozygous for the derived allele. One European landrace pig was heterozygous whereas all remaining European domestic pigs $(N=24)$ were homozygous for the derived allele. The derived allele is at a frequency of $40.00 \%$ in Asian wild boars and $11.36 \%$ in Asian domestic pigs population. Of 


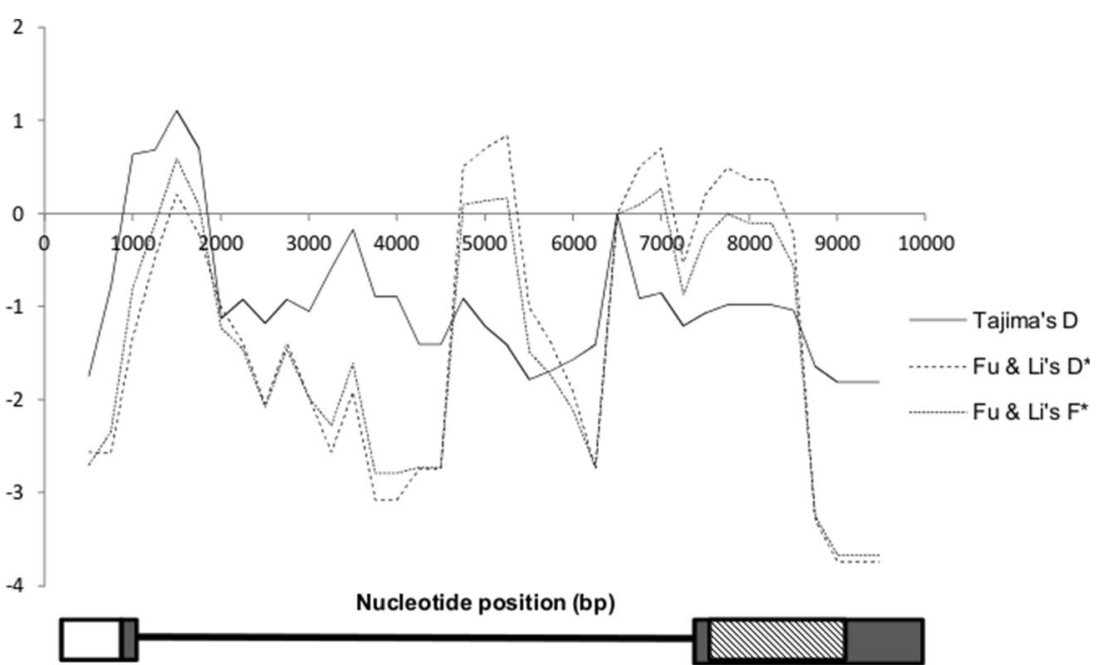

Fig. 1 Sliding window plots for TLR2 gene. Sliding window plots of Tajima's D, Fu and Li's F*, and Fu and Li's D* tests within the European (wild boars and domestic pigs combined) porcine population using window length of $1000 \mathrm{bp}$ and step size of $250 \mathrm{bp}$. The white box represents the five upstream regions, the thick black line represent the

the five Asian wild boars used in this study, one northern Chinese wild boar was homozygous for the derived allele, one northern Chinese wild boar and one Japanese wild boar were homozygous for the ancestral allele, whereas one southern Chinese wild boar and one northern Chinese wild boar were heterozygous. Seventeen Asian domestic pigs were homozygous for the ancestral allele. The remaining five Asian domestic pig breeds were heterozygous. Details on frequencies for TLR SNPs are as shown in Supplementary Table S3. Determination of the ancestral or derived state of an allele is described in the "Materials and methods" section. Figure 3 depicts a conservation of the G allele at TLR2 position 376 within three wild pig relatives. According to the strategy employed by Groenen et al. (2012), the G allele is the

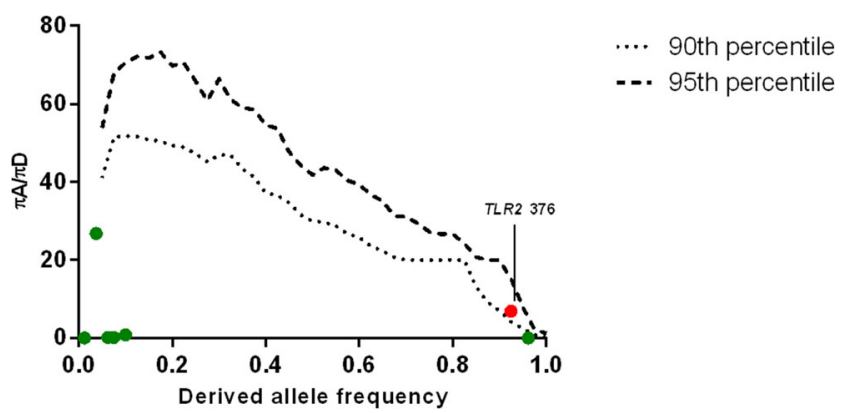

Fig. 2 Positive selection for TLR2. Detection of positive selection in European (wild boars and domestic pigs combined) porcine population TLR2 ectodomain using the DIND test. The short and long dashed lines represent the 90th and 95th percentiles of the empirical distribution of expected $\pi \mathrm{A} / \pi \mathrm{D}$ values obtained from 10,000 simulations of the ectodomain conditional on the number of segregating sites and the recombination rate of the ectodomain and integrating the wild boar demographic model previously described (Groenen et al. 2012). Green dots represent TLR2 SNPs under neutrality and red dot represents TLR2 SNPs under positive selection intronic region, the grey boxes show the exonic regions and the ectodomain is represented by the box with light down diagonal lines. The distal part of the ectodomain show significant values for neutrality indices

ancestral allele and the A allele is derived. The change from the ancestral to the derived allele at TLR2 SNP 376 is a nonsynonymous change (TLR2 SNP G376A, Ala126Thr) and is likely to affect protein function.

\section{Selective pressure differences between populations}

To determine whether selective pressures were heterogeneous between populations, $\mathrm{F}_{\mathrm{ST}}$ values for SNPs within TLR sequences were compared to the empirical distribution of $\mathrm{F}_{\mathrm{ST}}$ from SNPs obtained from noncoding genomic region sequences chosen to represent neutrally evolving regions (see "Materials and methods"). The rationale behind this approach was that differences in selective pressure between populations could lead to elevated levels of population differentiation at immune genes relative to neutrally evolving loci (Barreiro et al. 2009). The nonsynonymous variant TLR2 SNP G376A showed the highest level of population differentiation $\left(\mathrm{F}_{\mathrm{ST}}\right.$ between European domestic pigs and Asian domestic pigs $=0.86, p=0.02$ (Fig. 4a); $\mathrm{F}_{\mathrm{ST}}$ between European pigs (wild boars and domestic pigs combined) and Asian pigs (wild boars and domestic pigs combined) $=0.74, p=0.08$ (Fig. 4b)) A comparison of European wild boars and European domestic pigs indicated that TLR8 SNPs were highly differentiated relative to other TLR SNPs (Fig. 4c). However, the location of TLR8 gene on the $\mathrm{X}$ chromosome means it is prone to higher genetic drift which may result in elevated levels of population differentiation for TLR8 SNPs (Barreiro et al. 2009). F $_{\text {ST }}$ values between European wild boars and European domestic pigs were low relative to those between pigs of European and Asian origins, indicating weak differentiation between pig populations from the same geographic origin. Asian wild boar 


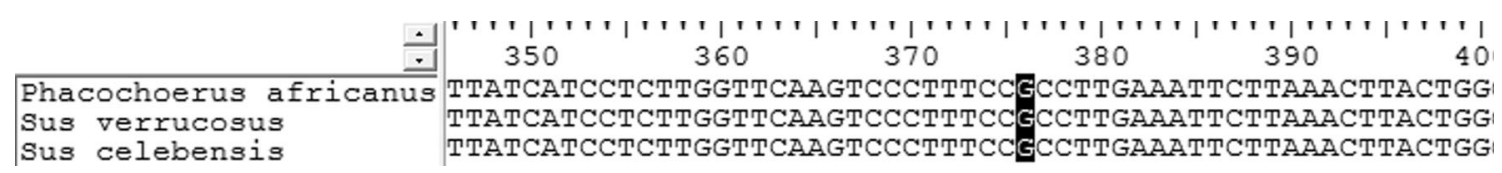

Fig. 3 Alignment of TLR2 sequences of Suidae species. Illustrated is the G allele at nucleotide position 376 within $T L R 2$ as the ancestral allele

population was not compared to any other population in terms of $F_{S T}$ given the small number of Asian wild boars involved in this study.

To determine whether positive selection drives the prevalence of certain $T L R$ haplotypes in European and Asian pig populations, haplotype reconstruction involving SNPs within the each TLR was done using the PHASE 2.1.1 software

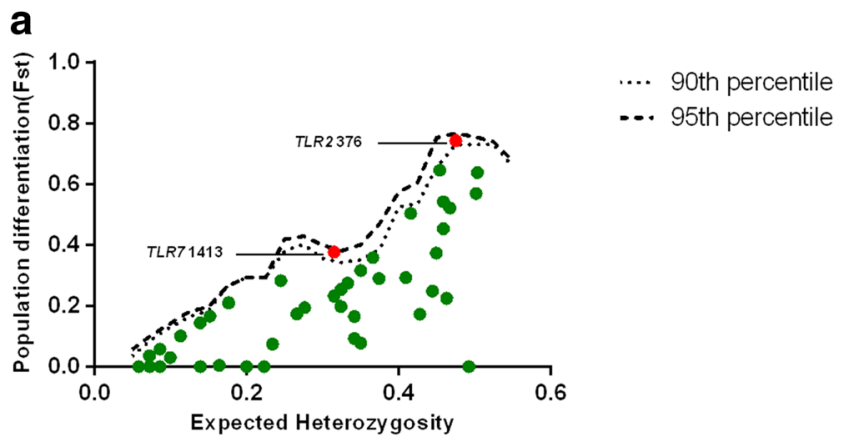

b

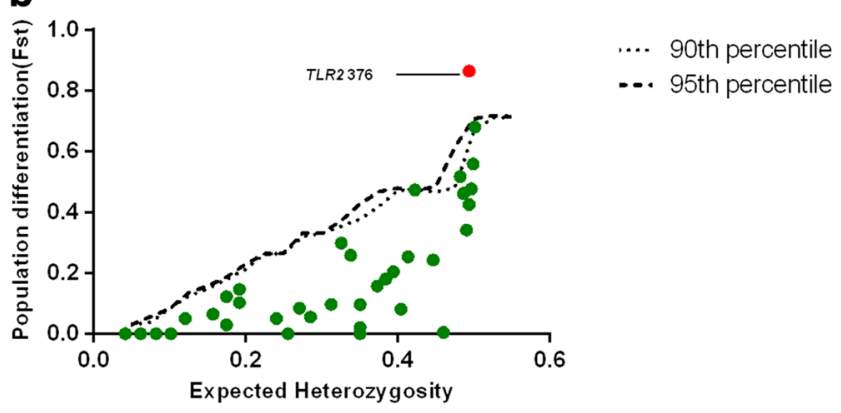

C

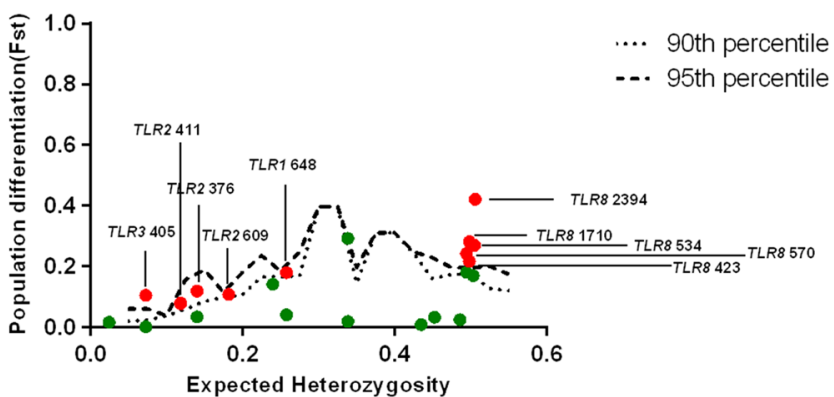

Fig. 4 Positive selection detection at TLR SNPs on the basis of population differentiation. $\mathrm{F}_{\mathrm{ST}}$ is plotted as a function of expected heterozygosity for every SNP between a Asian (wild boars and domestic pigs combined) versus European (wild boars and domestic pigs combined) porcine populations; b Asian domestic pig versus European domestic pig populations; and $\mathbf{c}$ European wild boar versus European domestic pig populations. Dots represent TLR SNPs. The short and long dashed lines represent the 90th and 95th percentiles of the empirical SNP distribution of $\mathrm{F}_{\mathrm{ST}}$ of The noncoding genomic region sequences
(Stephens and Scheet 2005) and the evolutionary relationships between haplotypes for each TLR were determined using a median-joining network. For TLR2, two high frequency haplotypes were observed and differed only at TLR2 SNP 376 (Fig. 5, Supplementary Table S4), where the high frequency haplotype $\left(\mathrm{H} \_1\right)$ dominated by the European individuals carried the derived SNP 376A allele and the high frequency haplotype $(\mathrm{H} 8)$ dominated by Asian individuals carried the ancestral SNP G376 allele. The high frequency haplotype within the European population was detected in all the European domestic pigs and the majority (10/15) of European wild boars. In order to estimate the divergence time of TLR2 sequences encoding the ectodomain and the age of TLR2 SNP G376A (Ala126Thr), maximum coalescence analysis by GENETREE (Griffiths and Tavare 1994) was utilized. Using all populations, the estimated time to most common ancestor $\left(\mathrm{T}_{\mathrm{MRCA}}\right)$ for the entire TLR2 sequences geneology was $0.900 \pm 0.28 \mathrm{Mya}$, which is close to the $\sim 1$ Mya since the split of the Asian and European wild boar, and the age of the 126Thr variant assuming neutrality was estimated to be $0.163 \pm 0.08$ Mya. The age of the derived allele and the presence of the haplotype carrying the derived allele in most European wild boars and all European domestic pigs involved in this study indicate that the allele arose within the wild boars, prior to the domestication process.

\section{Functional relevance of SNPs under positive selection}

To determine whether variants under selection are within functional domains of TLR receptors, variants were mapped onto TLR protein 3D structure. The TLR2 variant $126 \mathrm{Thr}$ is located within the N-terminal (on the fourth leucine-rich repeat (Supplementary Fig. S2)) and alpha helices of the TLR2 protein and is surface exposed (Fig. 6), indicating a likely role in protein-protein interactions. Porcine TLR2 amino acid sequence was also compared to human TLR2 amino acid sequence from Swiss-Prot to examine whether the site under positive selection was within functionally relevant domains of the protein. TLR2 variant $126 \mathrm{Thr}$ did not fall within any known region of functional region. Lastly, the TLR2 variation table in Ensembl was examined to determine the effects of substitutions at TLR2 amino acid site 126 on protein function. SIFT predicts whether an amino acid substitution affects protein function based on sequence homology and the physicochemical similarity between alternate amino acids (Kumar et al. 2009). Substitutions with scores $<0.05$ are called "deleterious" and those with scores $>0.05$ are called "tolerated." Within Ensembl, the substitution from Threonine to Alanine 
Fig. 5 Median-joining network for haplotypes at the TLR2 ectodomain. The circles represent haplotypes and the size of the circles are proportional to the frequency of the haplotypes. The mutation positions are shown along branches as red numbers. Asian wild boars, Asian domestic pigs, European wild boars, and European domestic pigs populations are shown in black, white, deep green, and light green colors, respectively. SNP 376, under positive selection is boxed and differentiates the Asian and European populations

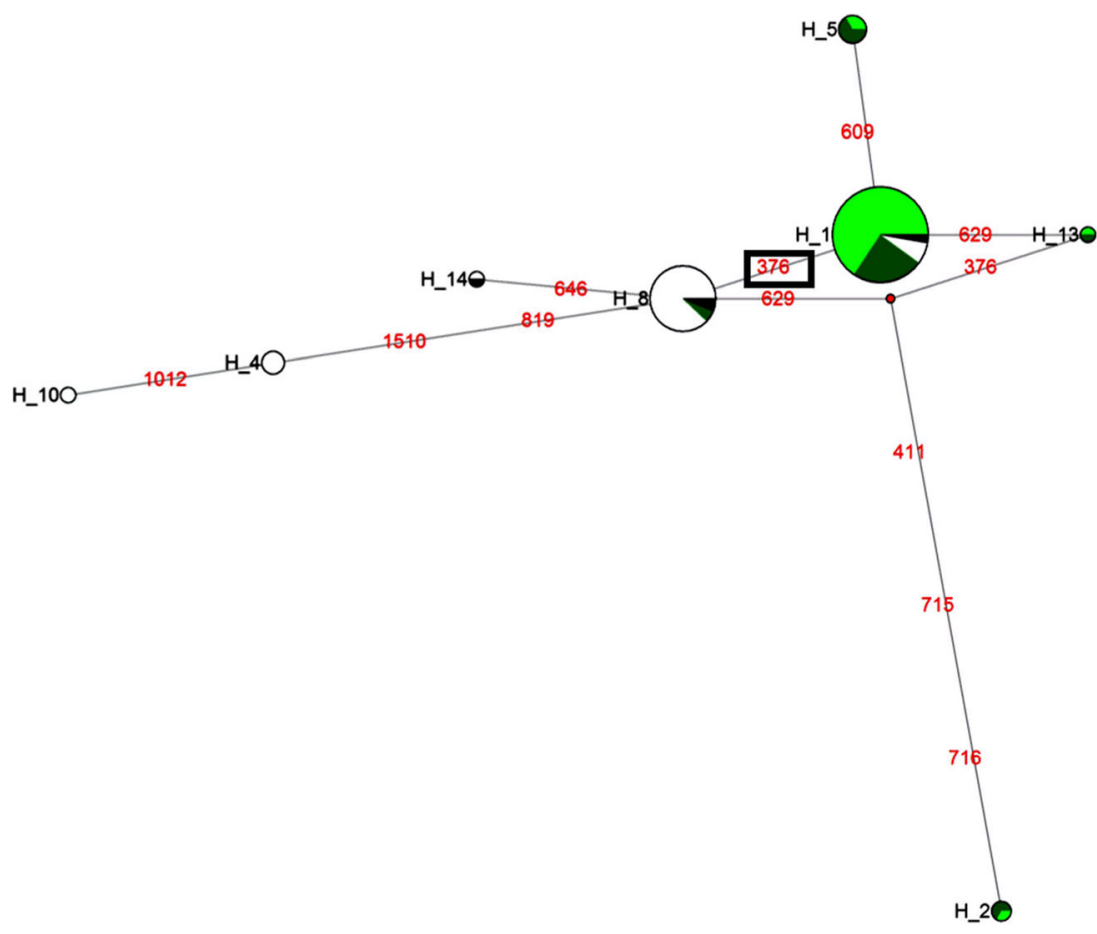

at porcine TLR2 126 (dbSNP identifier rs81218810) is predicted by SIFT to be deleterious (score $=0.03$ ), indicating that substitution at the TLR2 amino acid site 126 affects protein function.

\section{Discussion}

This study supports the hypothesis that geographically restricted selective pressures on European and Asian wild boars that have diverged over 1 Mya have resulted in genomic signatures of adaptation in porcine TLRs. Specifically, TLR2 SNP 376A (126Thr) of European pig (wild boars and domestic pigs combined) and not the Asian pig population showed evidence of positive selection, consistent with previous studies that have demonstrated that certain polymorphisms in TLR2 sequences encoding the ectodomain of primates (Takaki et al. 2012), wild rodents (Tschirren et al. 2013), and cattle (Jann et al. 2008) involved in pathogen recognition have evolved adaptively conferring selective advantage. Porcine $T L R 2$ is
Fig. 6 Location of amino acid residues under selection in TLR2 $3 \mathrm{D}$ protein structure. Amino acid residue TLR2 126Thr under positive selection within the European (wild boars and domestic pigs combined) porcine population is shown within the crystal structure of TLR1/TLR2 heterodimer (PDB ID 2Z7X) induced by binding of a triacylated lipopeptide

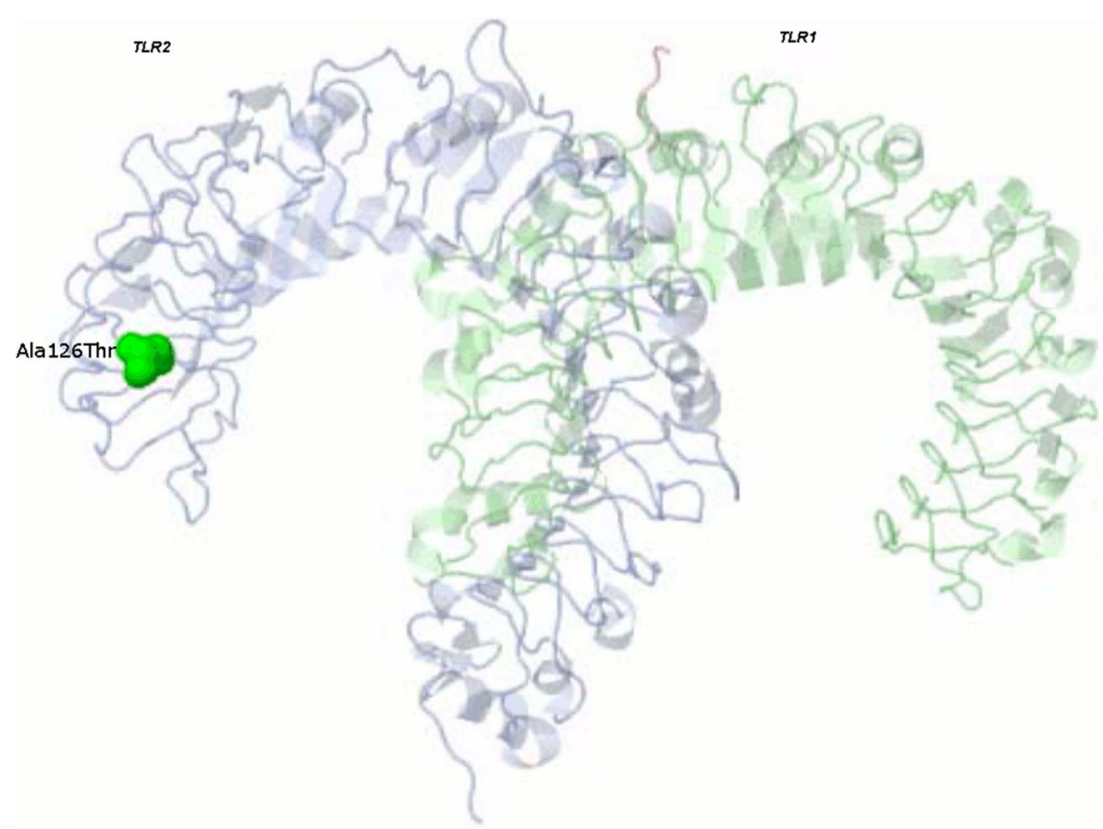


found at the distal end of the $\mathrm{q}$ arm of Chromosome 8, in a region with identified QTLs for some immune related traits (Jann et al. 2009), and is therefore a potential target for positive selection. Furthermore, the association of TLR2 with a wider panel of ligands and the need for heterodimerization (TLR2 forms heterodimers with TLR1 and TLR6 to recognize lipopeptide components of gram-positive and gram-negative bacterial cell walls) indicates that the $T L R 2$ region experiences contrasting evolutionary actions, including adaptive evolution to the environment and pathogens (Jann et al. 2008). A comparison of the genomic coordinates [8:79,824,541-79,834,592] of porcine TLR2 to recombination maps computed for four different pedigrees (Tortereau et al. 2012) indicate that Porcine $T L R 2$ lies in a region with a relatively low recombination rate on Chromosome 8. Regions with low recombination rate have been shown to be prone to positive selection (Barton 2000).

Within-population-based tests for positive selection utilized here have previously been employed to detect SNPs under positive selection in immune-related genes (interferons and Toll-like receptors) within European and Asian human populations (Barreiro et al. 2009; Manry et al. 2011). The positive selection of the derived allele TLR2 SNP 376A (126 Thr) in European pig population based on within population tests is likely due to selective pressure mediated by bacterial infectious agents encountered by the European wild boars following divergence from their Asian counterparts. Furthermore, the conservation of the ancestral allele across the three wild pig relatives, none of which have origins in the European continent, suggests that the derived allele has arisen in response to selective pressure pertaining to Europe. In humans, clinical genetic studies have indicated a role of TLR2 coding region polymorphisms in immune response to bacteria (Kang and Chae 2001; Schröder and Schumann 2005), demonstrating the action of selective pressure of bacterial origin on $T L R 2$ from a clinical perspective.

The estimated age of the TLR2 126Thr variant, its presence in European wild boars, European domestic pigs, Asian wild boars, and Asian domestic in this study, supports a situation where the selective pressure may have been of an ancient nature and present prior to the domestication process. The variant is likely to be of adaptive value to both European wild boars and their domesticated counterparts as they share some common bacterial agents (Meng et al. 2009), some of which may have persisted over extended periods. In this regard, one can expect that the selective pressure experienced by the European wild boars will persist in the domestic pigs. This may partly explain the high frequency of the derived allele in both European wild boars and domestic pigs. Widespread and long lasting gene have been reported between wild boars and domestic pigs across Eurasia (Chen et al. 2013; Frantz et al. 2015) and this can also explain the high frequency of the derived allele in both European wild boars and domestic pigs. Consistent with our observation of high frequency of a derived allele in both European wild boars and domestic pigs probably in response to pathogen-mediated selective pressure, genetic variability in wild boar populations has been detected to be preserved in local domestic breeds at genomic sites with potential phenotypic effects (Herrero-Medrano et al. 2014). The European domestic pig breeds involved in this study included both local and commercial breeds that have experienced artificial selective pressures of different intensities. For example, Charto Murciano, Cinta Senese, Cassertana, and Mangalitsa are local breeds and are not subjected to intense artificial selective pressures as experienced by commercial pigs like Pietrain, Duroc, and Landrace (Herrero-Medrano et al. 2014). These differences have however not resulted in much variation at the locus under positive selection as apart from one Landrace breed that was heterozygous, all other European domestic pigs were homozygous for the derived allele. Therefore, as mentioned earlier, the selective pressure responsible for the positive selective might have been in place before the domestication process. The estimated age of 163 , $000 \pm 80,000$ years of the derived allele under positive selection coincides with the late middle Pleistocene periods when there was an initiation of European wild boar expansion (about 190,000 years ago (Fang and Andersson 2006)) and when $S$. scrofa was spreading from southeastern to northeastern area of the Asian continent (about 140,000 to 253, 000 years ago (Watanobe et al. 2003)). This would have provided the necessary environment for the spread of infectious diseases that would lead to adaptation at host genes. Our analysis revealed high $\mathrm{F}_{\mathrm{ST}}$ values for certain $T L R$ alleles between European and Asian pig populations with the highest genetic differentiation detected for TLR2 SNP G376A (Ala126Thr). This may be due to different selective pressures associated with each population given that historically different continental populations have been exposed to different infectious agents (Troy et al. 2001; Novembre and Di Rienzo 2009). TLR2 mediates host immune response to gram-positive bacteria, and in the case of pigs, gram positive bacteria challenges peculiar to specific continents have been documented. For example, the methicillin-resistant Staphylococcus aureus sequence type (ST) 398 have been detected to be highly prevalent among pigs in Europe and North America whereas ST9 is predominant in Asia (Hasman et al. 2010; Asai et al. 2012; Jamrozy et al. 2012). Such heterogeneous selective pressures across populations can result in positive selection for resistance alleles in certain populations. A similar approach of genetic differentiation $\left(\mathrm{F}_{\mathrm{ST}}\right)$ between populations has been used to detect geographically restricted adaptation at type III interferons in European and Asian human populations (Manry et al. 2011). In a comparison of European and Chinese pig populations utilizing $\mathrm{F}_{\mathrm{ST}}$ outlier tests, the TLR $4 \mathrm{~g} .7485 \mathrm{C}$ have been shown to be under positive selection (Chen et al. 2013). Two haplotypes with highest frequencies are differentiated at TLR2 SNP 376 (Ala126 for major Asian haplotype and 
126 Thr for the major European haplotype), further supporting a possible role of positive selection at this site.

Even though the Swiss Prot database did not indicate that $T L R 2$ site 126 is within a functionally relevant region of the $T L R 2$ protein, the LRR4 within which TLR2 site 126 is located may be of functional relevance given that it contains TLR2 site 136, where amino acid substitution (Pro136Ala) is associated with the prevalence of pneumonia in pigs (Uenishi et al. 2011). Therefore, one can speculate that TLR2 Ala126Thr can be of medical relevance to porcine diseases. Nonsynonymous SNPs in LRR domains have been suggested to dramatically alter the ability of the molecule to identify extracellular pathogens (Fujita et al. 2003). The nonsynonymous nature of the TLR2 SNP G376A substitution, which causes a change of amino acid property from a nonpolar to a polar amino acid (Ala126Thr) suggests that the substitution may be important in adaptation of European pigs. The location of TLR2 126Thr within the alpha helices of the 3D structure of TLR1/TLR2 complex and at the N-terminal domain of the TLR2 protein suggests it is important for ligand detection for a variety of ligands including lipoteichoic acid and peptidoglycan (Mitsuzawa et al. 2001; Meng et al. 2003). The "deleterious" nature of the threonine to alanine substitution at TLR2 site 126 as predicted by SIFT further suggests this site is functionally relevant. A previous study investigating the effect of known polymorphisms in porcine TLRs on the recognition of Salmonella enterica serovar Choleraesusis (SC) did not implicate TLR2 SNP G376A in attenuating responses to SC (Shinkai et al. 2011). However, recognition of other pathogens known to affect pigs may be affected by TLR2 SNP G376A and thus requires further investigation.

Results presented here suggests a role of pathogenmediated selective pressures among pig populations in driving the differentiation at TLR2 SNP G376A (Ala126Thr). Future experimental functional analyses are required to determine how such SNP variant affect porcine immune response. A recent study (Tschirren et al. 2013) involving a wild rodent population has identified an association between Borrelia infection and haplotypes carrying the variants Ala and Thr (TLR2 Thr276Ala) located within the ectodomain (Tschirren et al. 2013). The study of Tschirren et al. (2013) thus has demonstrated a role of alanine-threonine substitutions within $T L R 2$ in infectious diseases.

\section{Conclusions}

In conclusion, this study provides evidence, based on within and between population tests, that European wild boars and domestic pigs show evidence of adaptation which is reflected in TLR2 as signatures of selection, whereas no such evidence was observed in Asian wild boars and domestic breeds. Thus, our study suggests that TLR2 126Thr present in European wild boars, European domestic pigs, Asian wild boars, and Asian domestic pigs has evolved under positive selection within the European pigs involved in this study, probably in response to pathogen-mediated selective pressures. Experimental studies designed to investigate the role of the TLR2 126Thr in ligand binding and subsequent immune response are needed.

Acknowledgments We are thankful to Dr. Laurie Rund of the Animal Sciences department of University of Illinois and Dr. Greger Larson of the School of Archaeology of Oxford University for reading the manuscript and making useful suggestions. We are grateful to J. M. Herrero-Medrano of the Wageningen University for providing mitochondrial D-loop sequences. We are also thankful to Dr. Guillaume Laval of the Unit of Human Evolutionary Genetics, Centre National de la Recherche Scientific, Paris, France, for providing scripts for the detection of $\mathrm{F}_{\mathrm{ST}}$ outliers and DIND test. This work was funded by US Department of Agriculture (USDA) Agriculture Research Service (ARS) Grant 58-5438-2-307 to LBS.

Open Access This article is distributed under the terms of the Creative Commons Attribution 4.0 International License (http:// creativecommons.org/licenses/by/4.0/), which permits unrestricted use, distribution, and reproduction in any medium, provided you give appropriate credit to the original author(s) and the source, provide a link to the Creative Commons license, and indicate if changes were made.

\section{References}

Akira S, Uematsu S, Takeuchi O (2006) Pathogen recognition and innate immunity. Cell 124:783-801

Amaral AJ, Ferretti L, Megens H-J et al (2011) Genome-wide footprints of pig domestication and selection revealed through massive parallel sequencing of pooled DNA. PLoS One 6:e14782

Asai T, Hiki M, Baba K et al (2012) Presence of Staphylococcus aureus ST398 and ST9 in swine in Japan. Jpn J Infect Dis 65:551-552

Barreiro LB, Ben-Ali M, Quach H et al (2009) Evolutionary dynamics of human Toll-like receptors and their different contributions to host defense. PLoS Genet 5:e1000562. doi:10.1371/journal.pgen. 1000562

Barton NH (2000) Genetic hitchhiking. Philos Trans R Soc Lond B Biol Sci 355:1553-1562

Bergman I-M, Rosengren JK, Edman K, Edfors I (2010) European wild boars and domestic pigs display different polymorphic patterns in the Toll-like receptor (TLR) 1, TLR2, and TLR6 genes. Immunogenetics 62:49-58

Bonfield JK, Smith KF, Staden R (1995) A new DNA sequence assembly program. Nucleic Acids Res 23:4992-4999

Bosse M, Megens H-J, Madsen O et al (2012) Regions of homozygosity in the porcine genome: consequence of demography and the recombination landscape. PLoS Genet 8:e1003100

Chen S, Gomes R, Costa Vet al (2013) How immunogenetically different are domestic pigs from wild boars: a perspective from singlenucleotide polymorphisms of 19 immunity-related candidate genes. Immunogenetics 65:737-748

Dereeper A, Nicolas S, Le Cunff L et al (2011) SNiPlay: a web-based tool for detection, management and analysis of SNPs. Application to grapevine diversity projects. BMC Bioinforma 12:134 
Excoffier L, Lischer HEL (2010) Arlequin suite ver 3.5: a new series of programs to perform population genetics analyses under Linux and Windows. Mol Ecol Resour 10:564-567

Excoffier L, Dupanloup I, Huerta-Sánchez E et al (2013) Robust demographic inference from genomic and SNP data. PLoS Genet 9: e1003905

Fang M, Andersson L (2006) Mitochondrial diversity in European and Chinese pigs is consistent with population expansions that occurred prior to domestication. Proc Biol Sci 273:1803-1810

Frantz LAF, Schraiber JG, Madsen O et al (2013) Genome sequencing reveals fine scale diversification and reticulation history during speciation in Sus. Genome Biol 14:R107. doi:10.1186/gb-2013-14-9-r107

Frantz LAF, Schraiber JG, Madsen O, et al (2015) Evidence of long-term gene flow and selection during domestication from analyses of Eurasian wild and domestic pig genomes. Nat Genet 47:1141-1148

Fujita M, Into T, Yasuda M et al (2003) Involvement of leucine residues at positions 107,112 , and 115 in a leucine-rich repeat motif of human Toll-like receptor 2 in the recognition of diacylated lipoproteins and lipopeptides and Staphylococcus aureus peptidoglycans. J Immunol 171:3675-3683

Giuffra E, Kijas JM, Amarger V et al (2000) The origin of the domestic pig: independent domestication and subsequent introgression. Genetics 154:1785-1791

Griffiths RC, Tavare S (1994) Ancestral inference in population genetics. Stat Sci 9:307-319

Groenen MAM, Archibald AL, Uenishi H et al (2012) Analyses of pig genomes provide insight into porcine demography and evolution. Nature 491:393-398

Hasman H, Moodley A, Guardabassi L et al (2010) Spa type distribution in Staphylococcus aureus originating from pigs, cattle and poultry. Vet Microbiol 141:326-331. doi:10.1016/j.vetmic.2009.09.025

Herrero-Medrano JM, Megens H-J, Groenen MAM et al (2014) Wholegenome sequence analysis reveals differences in population management and selection of European low-input pig breeds. BMC Genomics 15:601

Jamrozy DM, Fielder MD, Butaye P, Coldham NG (2012) Comparative genotypic and phenotypic characterisation of methicillin-resistant Staphylococcus aureus ST398 isolated from animals and humans. PLoS One 7:e40458. doi:10.1371/journal.pone.0040458

Jann OC, Werling D, Chang J-S et al (2008) Molecular evolution of bovine Toll-like receptor 2 suggests substitutions of functional relevance. BMC Evol Biol 8:288

Jann OC, King A, Corrales NL et al (2009) Comparative genomics of Toll-like receptor signalling in five species. BMC Genomics 10:216

Kang T-J, Chae G-T (2001) Detection of Toll-like receptor 2 (TLR2) mutation in the lepromatous leprosy patients. FEMS Immunol Med Microbiol 31:53-58

Kumar P, Henikoff S, Ng PC (2009) Predicting the effects of coding nonsynonymous variants on protein function using the SIFT algorithm. Nat Protoc 4:1073-1081

Larson G, Dobney K, Albarella U et al (2005) Worldwide phylogeography of wild boar reveals multiple centers of pig domestication. Science 307:1618-1621

Lazarus R, Vercelli D, Palmer LJ et al (2002) Single nucleotide polymorphisms in innate immunity genes: abundant variation and potential role in complex human disease. Immunol Rev 190:9-25

Leulier F, Lemaitre B (2008) Toll-like receptors - taking an evolutionary approach. Nat Rev Genet 9:165-178

Librado P, Rozas J (2009) DnaSP v5: a software for comprehensive analysis of DNA polymorphism data. Bioinformatics 25:1451-1452

Luetkemeier ES, Sodhi M, Schook LB, Malhi RS (2010) Multiple Asian pig origins revealed through genomic analyses. Mol Phylogenet Evol 54:680-686

Manry J, Laval G, Patin E et al (2011) Evolutionary genetic dissection of human interferons. J Exp Med 208:2747-2759
Megens H-J, Crooijmans RPMA, San Cristobal M et al (2008) Biodiversity of pig breeds from China and Europe estimated from pooled DNA samples: differences in microsatellite variation between two areas of domestication. Genet Sel Evol 40:103-128

Meng G, Grabiec A, Vallon M et al (2003) Cellular recognition of tri-/dipalmitoylated peptides is independent from a domain encompassing the N-terminal seven leucine-rich repeat (LRR)/LRR-like motifs of TLR2. J Biol Chem 278:39822-39829

Meng XJ, Lindsay DS, Sriranganathan N (2009) Wild boars as sources for infectious diseases in livestock and humans. Philos Trans R Soc Lond B Biol Sci 364:2697-2707

Mitsuzawa H, Wada I, Sano H et al (2001) Extracellular Toll-like receptor 2 region containing Ser40-Ile64 but not Cys30-Ser39 is critical for the recognition of Staphylococcus aureus peptidoglycan. J Biol Chem 276:41350-41356

Morozumi T, Uenishi H (2009) Polymorphism distribution and structural conservation in RNA-sensing Toll-like receptors 3, 7, and 8 in pigs. Biochim Biophys Acta 1790:267-274

Niknafs N, Kim D, Kim R et al (2013) MuPIT interactive: webserver for mapping variant positions to annotated, interactive 3D structures. Hum Genet 132:1235-1243

Novembre J, Di Rienzo A (2009) Spatial patterns of variation due to natural selection in humans. Nat Rev Genet 10:745-755

OIE (2012) W organisation for animal health. General disease information sheets. http:/www.oie.int/fileadmin/Home/eng/Media_Center/ docs/pdf/Disease_cards/

Rubin C-J, Megens H-J, Martinez Barrio A et al (2012) Strong signatures of selection in the domestic pig genome. Proc Natl Acad Sci U S A 109:19529-19536

Schröder NWJ, Schumann RR (2005) Single nucleotide polymorphisms of Toll-like receptors and susceptibility to infectious disease. Lancet Infect Dis 5:156-164

Shinkai H, Tanaka M, Morozumi T et al (2006) Biased distribution of single nucleotide polymorphisms (SNPs) in porcine Toll-like receptor 1 (TLR1), TLR2, TLR4, TLR5, and TLR6 genes. Immunogenetics 58:324-330

Shinkai H, Suzuki R, Akiba M et al (2011) Porcine Toll-like receptors: recognition of Salmonella enterica serovar Choleraesuis and influence of polymorphisms. Mol Immunol 48:1114-1120

Stephens M, Scheet P (2005) Accounting for decay of linkage disequilibrium in haplotype inference and missing-data imputation. Am J Hum Genet 76:449-462

Takaki A, Yamazaki A, Maekawa Tet al (2012) Positive selection of Tolllike receptor 2 polymorphisms in two closely related old world monkey species, rhesus and Japanese macaques. Immunogenetics 64:15-29

Tamura K, Peterson D, Peterson N et al (2011) MEGA5: molecular evolutionary genetics analysis using maximum likelihood, evolutionary distance, and maximum parsimony methods. Mol Biol Evol 28: 2731-2739

Thompson JD, Gibson TJ, Plewniak F et al (1997) The CLUSTAL X windows interface: flexible strategies for multiple sequence alignment aided by quality analysis tools. Nucleic Acids Res 25:48764882

Tortereau F, Servin B, Frantz L et al (2012) A high density recombination map of the pig reveals a correlation between sex-specific recombination and GC content. BMC Genomics 13:586

Troy CS, MacHugh DE, Bailey JF et al (2001) Genetic evidence for NearEastern origins of European cattle. Nature 410:1088-1091

Tschirren B, Andersson M, Scherman K et al (2013) Polymorphisms at the innate immune receptor TLR2 are associated with Borrelia infection in a wild rodent population. Proc Biol Sci 280:20130364

Uenishi H, Shinkai H, Morozumi T et al (2011) Polymorphisms in pattern recognition receptors and their relationship to infectious disease susceptibility in pigs. BMC Proc 5(Suppl 4):S27 
Ursing BM, Arnason U (1998) Analyses of mitochondrial genomes strongly support a hippopotamus-whale clade. Proc Biol Sci 265: 2251-2255

Wagh K, Bhatia A, Alexe G et al (2012) Lactase persistence and lipid pathway selection in the Maasai. PLoS One 7:e44751

Watanobe T, Ishiguro N, Nakano M (2003) Phylogeography and population structure of the Japanese wild boar Sus scrofa leucomystax: mitochondrial DNA variation. Zool Sci 20:1477-1489
Werling D, Jungi TW (2003) TOLL-like receptors linking innate and adaptive immune response. Vet Immunol Immunopathol 91:1-12

Zimmerman J, Karriker L, Ramirez A et al (eds) (2012) Disease transmission and biosecurity. In: Diseases of swine, 10th edn. WileyBlackwell, Hoboken, pp. 141-164 